\title{
UNE NOUVELLE FILAIRE \\ DE LA PIE, EUFILARIA KALIFAI N. SP. (LEMDANINAE) et son développement chez Culicoides nubeculosus
}

\author{
P. MILLET et O. BAIN*
}

RÉSUMÉ. E. kalifai n. sp. est décrit chez Pica pica galliae capturée en France (Hérault); cette espèce est proche d'E. longicaudata des Corvidae d'Amérique du Nord, ainsi que d'E. delicata et $E$. bartlettae des Turdidae d'Europe, mais s'en distingue principalement par la microfilaire de grande taille $(180-220 \mu \mathrm{m})$. Le cycle d'E. kalifai est réalisé chez Culicoides nubeculosus. Ce groupe de 4 espèces d'Eufilaria est très homogène, mais l'extrémité caudale des formes infestantes est ornée de 4 languettes chez les parasites de Corvidae; elle est lisse chez ceux de Turdidae.

\section{A new Filaria of the magpie Eufilaria kalifai n. sp. (Lemdaninae) and its development in Culicoides nubeculosus}

SUMMARY. E. kalifai n. sp. (Lemdaninae) is described from Pica pica galliae captured in France (Herault); this species resembles E. longicaudata from North American Corvidae as well as E. delicata and E. bartlettae from European Turdidae but is distinguished principally by the large size of the microfilaria $(180-220 \mu \mathrm{m})$. The life is completed using Culicoides nubeculosus as intermediate host. The above four species of Eufilaria are very similar but the caudal extremity of the infective stage has four "languettes" in the parasites of Corvidae ; it is smooth in parasites of Turdidae.

Des observations faites sur une quinzaine de pies, Pica pica galliae Kleinschmidt, capturées dans l'Hérault en janvier-février 1983, ont révélé une fréquence élevée du parasitisme par les Filaires.

Trois espèces se rencontrent, seules ou associées : Pseudlemdana corvicola (Schikhobalowa, 1948) décrite en URSS et connue jusqu'à présent uniquement chez les Corvidés de cette région; Chandlerella sp., peu fréquente, dont nous n'avons que 2 femelles; une nouvelle Eufilaria, E. kalifai n. sp., que nous décrivons ici.

Le stade infestant de cette nouvelle espèce, obtenu au laboratoire chez Culicoïdes nubeculosus, est comparé aux autres formes larvaires connues dans le genre : une espèce parasite de Corvidés en Amérique du Nord et deux espèces parasites de Turdidés en Europe.

* Laboratoire des Vers, associé au C.N.R.S., Muséum National d'Histoire Naturelle, 61 rue Buffon, F 75231 Paris Cedex 05.

I. Ce travail a été effectué grâce à une subvention de l'Organisation Mondiale de la Santé.

Accepté le 8 juillet 1983 . 


\section{Modèle biologique}

Les pies parasitées par Eufilaria kalifai $\mathrm{n}$. sp. ont deux origines :

1) Pérols (au sud de Montpellier). 5 pies positives sur 9 observées (infections monospécifiques), $\mathrm{n}^{\circ}$ des pies : 7, 8, 10, 13 et $20 \mathrm{RC}$.

2) Teyran (dans le nord-est de l'Hérault). 2 pies positives sur 5 observées, l'une associée avec $P$. corvicola (n 5 RC), l'autre avec cette espèce plus Chandlerella sp. (no 3 RC).

Les pies $7 \mathrm{RC}, 8 \mathrm{RC}$ et $20 \mathrm{RC}$ sont maintenues en captivité ; elles permettent l'étude de la microfilarémie et la réalisation du cycle larvaire chez les Culicoides nubeculosus élevés au laboratoire.

Les 4 autres pies ont été autopsiées et des adultes d'Eufilaria ont été trouvés chez 3 d'entre elles; ils sont tous situés sous la peau :

$\mathrm{n}^{\circ} 10 \mathrm{RC}: 1$ o holotype et 1 ô allotype, situés dans la région ventrale;

no $3 \mathrm{RC}: 2$ ㅇ situées respectivement à la cuisse et à la base du cou ;

no $5 \mathrm{RC}: 1$ ô à la base du cou. La pie $5 \mathrm{RC}$ n'a pas de microfilaire d'Eufilaria.

\section{I - Description d'Eufilaria kalifai n. sp.}

Cuticule légèrement gonflée et festonnée dans la région antérieure, sur les faces ventrale et dorsale, finement striée sur le reste du corps. Cordes latérales très épaisses et relativement larges, à 3 files de noyaux. Tête arrondie suivie d'un léger rétrécissement. Disposition et nombre des papilles de la tête indiqués sur les figures $1 C, D, E$ et $2 B$ et $C$; chaque amphide s'ouvre par une fente allongée en avant de laquelle se trouve un relief cuticulaire qui correspond vraisemblablement à une papille labiale externe latérale vestigiale ; 2 plis cuticulaires, un ventral et un dorsal, s'incurvant vers la bouche, joignent les papilles céphaliques.

Bouche très réduite mais nettement aplatie latéralement. Esophage très mince et non divisé ; l'intestin forme, chez certains spécimens un caecum qui remonte le long de l'œsophage sur une longueur de $100-150 \mu \mathrm{m}$ et est appliqué contre lui ( $f \mathrm{~g} g .1 \mathrm{~F}$ ).

Femelle (fig. 1).

Vulve un peu en arrière de l'œsophage. Ovéjecteur sans sphincter complexe comprenant un vagina vera court suivi d'un vagina uterina droit, dirigé vers l'arrière se divisant à moins de $1 \mathrm{~mm}$ de la vulve. Tubes génitaux peu contournés; opisthodelphie ; apex des utérus remplis de spermatozoïdes. Queue de forme et de longueur assez variable mais régulièrement ornée par 2 bosses latéro-ventrales arrondies; la queue de la + holotype porte une vésicule cuticulaire impossible à détacher du corps qui est probablement un reste d'exuvie de la mue IV, non expulsée chez ce spécimen.

Dimensions : $q$ holotype : corps long de $18 \mathrm{~mm}$ et large de $130 \mu \mathrm{m}$; anneau nerveux à $170 \mu \mathrm{m}$ de l'apex; œsophage long de $370 \mu \mathrm{m}$; vulve à $570 \mu \mathrm{m}$ de l'apex ; 


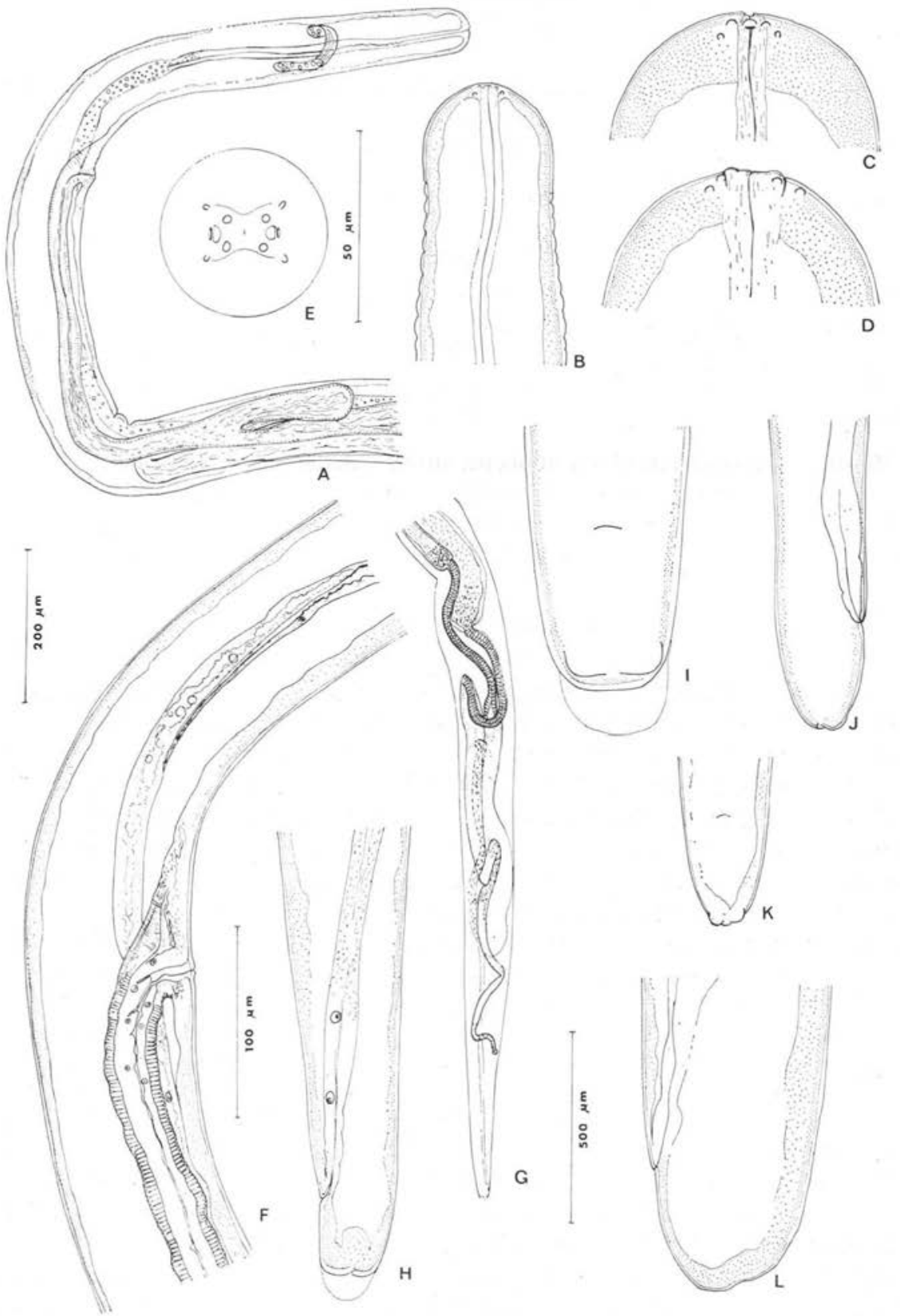

FIG. I. - Eufilaria kalifai n. sp., ᄋ; A, F, G, H, I, holotype ; A : région antérieure, vue latérale ; $\mathrm{B}$ : région céphalique, vue latérale; $\mathrm{C}, \mathrm{D}, \mathrm{E}$ : tête, vues latérale, médiane et apicale ; F : fin de l'œsophage (et caecum intestinal) et début de l'ovéjecteur, vue latérale ; G : région postérieure et appareil génital, vue latérale; $\mathrm{H}$ et $\mathrm{I}$ : région caudale en vue latérale et ventrale : J et $\mathrm{K}: i d e m, 2^{\mathrm{e}}$ 우 $\mathrm{L}: i d e m, 3^{\mathrm{e}}$ ․ (A, éch. $200 \mu \mathrm{m} ; \mathrm{C}, \mathrm{D}, \mathrm{E}, \mathrm{I}, \mathrm{L}$, éch. $50 \mu \mathrm{m} ; \mathrm{B}, \mathrm{F}, \mathrm{H}, \mathrm{J}$, $\mathrm{K}$, éch. $100 \mu \mathrm{m}$; G, éch. $500 \mu \mathrm{m}$.) 
vagina vera long de $20 \mu \mathrm{m}$; division de l'ovéjecteur à $540 \mu \mathrm{m}$ de la vulve ; ovaires à 380 et $1130 \mu \mathrm{m}$ de l'extrémité caudale; queue longue de $36 \mu \mathrm{m}$.

2 o du lot $3 \mathrm{RC}$ : corps long de 22 et $23 \mathrm{~mm}$, large de 110 et $115 \mu \mathrm{m}$; anneau nerveux à 170 et $200 \mu \mathrm{m}$ de l'apex; vulve à 650 et $710 \mu \mathrm{m}$ de l'apex; œsophage long de 350 et $430 \mu \mathrm{m}$; ovéjecteur long de 350 et $950 \mu \mathrm{m}$; queue longue de 52 et $30 \mu \mathrm{m}$.

Mâle (fig. 2).

Cinq papilles caudales ( 3 et 2) disposées sur 2 saillies cuticulaires latéro-ventrales ; présence d'une bosse arrondie en avant de l'ouverture cloacale, sans papille perceptible; ouverture cloacale grande, en arc de cercle; queue très courte, avec un léger mamelon terminal et 2 phasmides latéro-dorsales. Spicule gauche un peu plus large et long que le droit, avec une torsion en spirale bien marquée à mi-longueur et terminé par une pointe conique étroite. Spicule droit avec un talon dorsal subterminal et une extrémité obtuse.

Dimensions : $\sigma^{\hat{t}}$ allotype : corps long de $8,5 \mathrm{~mm}$, large de $75 \mu \mathrm{m}$; anneau nerveux à $160 \mu \mathrm{m}$ de l'apex; œsophage long de $450 \mu \mathrm{m}$; début du testicule à $550 \mu \mathrm{m}$ de la tête ; queue longue de $8 \mu \mathrm{m}$; spicules gauche et droit longs de 60 et $55 \mu \mathrm{m}$.

\section{Microfilaire (fig. $3 A$ et $4 A, B, C, D, J, K$ )}

Pas de gaine. Tête un peu renflée à frais, aussi large que le corps après fixation, armée par deux formations cuticulaires très nettes : d'un côté le crochet triangulaire, et du côté opposé un arc cuticulaire à petite pointe terminale; à frais le crochet apparaît très saillant, un peu comme un bec d'oiseau ; au giemsa, on n'observe plus qu'une petite saillie apicale. Espace céphalique grand. Pore excréteur souvent souligné par une sécrétion saillante. Corps interne deux fois plus long que large, souvent pincé à mi-longueur. Grande cellule R1, de forme rectangulaire et au cytoplasme développé, souvent visible au Giemsa ; R2-R3 à mi-distance entre R1 et R4 ou formant un groupe avec R4. Queue longue, avec une pointe caudale allongée à portion terminale anucléée fine, terminée en très léger bouton ou simplement effilée.

\section{Dimensions :}

1) Microfilaires utérines des $q$ fixées à l'alcool chaud : chez la femelle holotype, 120$145 \mu \mathrm{m}$ de long sur $4 \mu \mathrm{m}$ de large avec corps interne long de $8 \mu \mathrm{m}$; chez une des femelles $3 \mathrm{RC}$ corps longs de $145 \mu \mathrm{m}$ à $155 \mu \mathrm{m}$ et larges de $4 \mu \mathrm{m}$; dans les deux cas les microfilaires utérines sont plissées et sinueuses et leurs dimensions sous-estimées.

2) Microfilaires sanguines immobilisées à la chaleur ou colorées vitalement :

— pie $10 \mathrm{RC}$ : corps longs de 193 à $198 \mu \mathrm{m}$ et larges de 4,5 $\mu \mathrm{m}$; corps interne long de $12 \mu \mathrm{m}$;

— pie $20 \mathrm{RC}$ : corps longs de 195 à $220 \mu \mathrm{m}$; pour une microfilaire longue de $195 \mu \mathrm{m}$ et large de $5 \mu \mathrm{m}$, par exemple, noyau excréteur, corps interne, R1 et R4 respectivement à $62 \mu \mathrm{m}, 73 \mu \mathrm{m}, 115 \mu \mathrm{m}, 135 \mu \mathrm{m}$ et $167 \mu \mathrm{m}$ de l'apex ; corps interne long de $10 \mu \mathrm{m}$. 

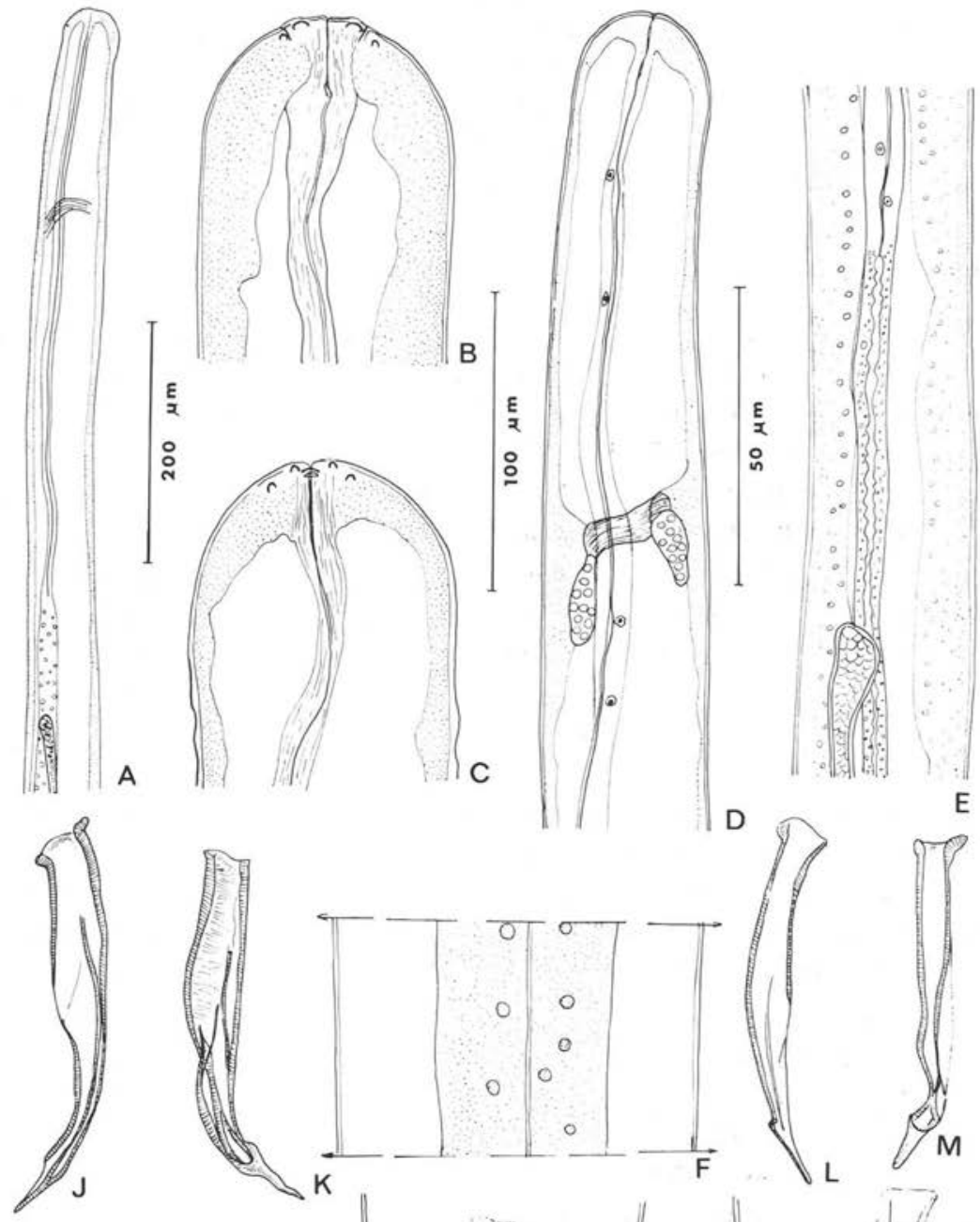

$\mathrm{E}$
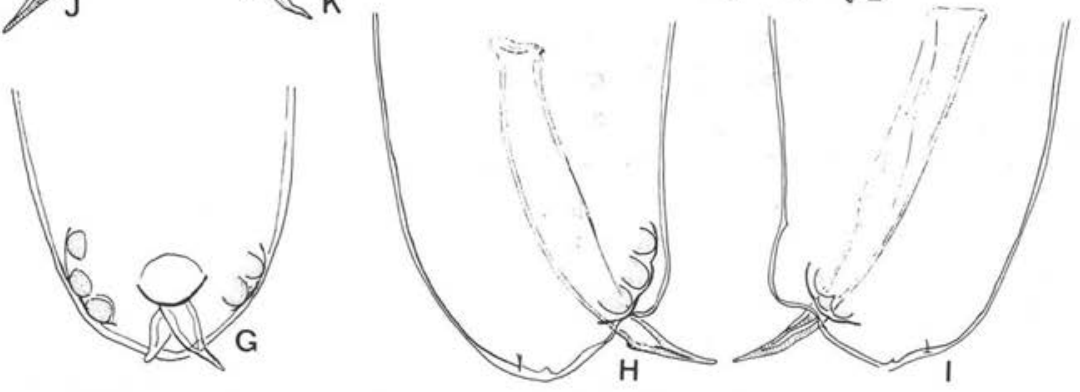

FIG. 2. - Eufilaria kalifai $\mathrm{n}$. sp., ô allotype ; A : région antérieure, vue latérale ; B et C : région apicale, vues médiane et latérale; D : structure de l'œsophage, vue latérale ; E : jonction œsophage-intestin et naissance du testicule, vue médiane ; F : corde latérale, vue latérale ; G, H, I : queue en vues ventrale, latérale droite et latérale gauche; J et K : spicule gauche, en vue latérale et subventrale; $L$ et $M$ : spicule droit en vue latérale et subventrale. (A, éch. $200 \mu \mathrm{m}$; B, C, G, H, I, J, K, L, M, éch. $50 \mu \mathrm{m}$; D, E, F, éch. 100 $\mu \mathrm{m}$.) 
3) Microfilaires sanguines en gouttes épaisses colorées au Giemsa (20 RC, $7 \mathrm{RC}$ et $8 \mathrm{RC}$ ) : corps longs de 158 à $180 \mu \mathrm{m}$ et larges de $5 \mu \mathrm{m}$; pour une microfilaire de $167 \mu \mathrm{m}$ de long, espace céphalique long de $6 \mu \mathrm{m}$; anneau nerveux, pore excréteur, corps interne, R1 et pore anal à $37 \mu \mathrm{m}, 52 \mu \mathrm{m}, 90 \mu \mathrm{m}, 110 \mu \mathrm{m}$ et $132 \mu \mathrm{m}$ de l'apex ; corps interne long de $9 \mu \mathrm{m}$; pointe caudale anucléée longue de $7 \mu \mathrm{m}$.

\section{Discussion}

Selon Anderson et Bain (1976), le genre Eufilaria Seurat, 1921 (= Eufilariella Sonin, $1965 ;=$ Neofilaria Deshmukh, 1968) groupe les Filaires vivipares d'Oiseaux ayant un anus subterminal chez le mâle, des spicules subégaux et un œesophage non divisé et court. Les spécimens de la pie se placent aisément dans ce genre.

Il comprend actuellement les 10 espèces analysées récemment par Bartlett (1979), plus une espèce décrite ultérieurement (Bain, 1980).

L'espèce type, Eufilaria sergenti Seurat, 1921, parasite de Ploceidae en Afrique, et E. capsulata (Annett, Dutton et Elliot, 1901), parasite de Pycnonotidae et Ploceidae, en Afrique également, sont bien différentes de notre matériel : ce sont des Filaires larges (plus de $100 \mu \mathrm{m}$ chez les $\widehat{\sigma}$ et de $300 \mu \mathrm{m}$ chez les ) $)$ à vulve située en avant de la jonction œsophage-intestin et l'orifice cloacal n'est pas réellement subterminal (respectivement à 80 et $110 \mu \mathrm{m}$ de l'extrémité caudale).

Les autres espèces d'Eufilaria forment un second groupe n'ayant pas ces caractères et sont plus proches de nos spécimens.

E. singhi (Sultana, 1961), parasite de Campephagidae en Indes, a un œsophage divisé et relativement long $(680-880 \mu \mathrm{m})$; le spicule droit est nettement plus court que le gauche (respectivement 39 et $64 \mu \mathrm{m}$ ).

E. alii (Deshmukh, 1968), parasite de Turnicidae, et E. buckleyi (Deshmukh, 1968), parasite de Phasianidae en Indes, ainsi qu'E. coua Anderson et Prestwood, 1969, parasite de Cuculidae à Madagascar, ont 2 à 3 paires de papilles précloacales en plus des papilles portées par les 2 bosses cuticulaires paracloacales ; de plus les microfilaires sont de petite taille (inférieure à $100 \mu \mathrm{m}$ de long).

E. macintoshi Anderson et Bennett, 1960, parasite de Ploceidae d'Indonésie, a une queue très courte chez la ㅇ $(15 \mu \mathrm{m})$, des papilles disposées de façon asymétrique sur la tête, et la microfilaire, connue seulement par la forme sanguine, est courte $(108 \mu \mathrm{m})$, à gaine et à queue entièrement nucléée, arrondie à l'extrémité.

E. asiatica Singh, 1949, parasite de Corvidae en Indes, et connu seulement par le $\widehat{\delta}$, a des spicules de grande taille (113-114 $\mu \mathrm{m}$ et $119-124 \mu \mathrm{m})$.

Trois espèces sont particulièrement proches de notre matériel ; ce sont les microfilaires qui fournissent le caractère le plus sûr pour les différencier.

E. longicaudata Hibler, 1963, parasite de Corvidae en Amérique du Nord et E. bartlettae Bain, 1980, parasite de Turdidae en Europe, ont des microfilaires courtes (respectivement $91-110 \mu \mathrm{m}$ et $110-122 \mu \mathrm{m}$ ). 
E. delicata Supperer, 1958, parasite de Turdidae $^{1}$ en Europe, a une microfilaire dont la taille se rapproche de celle de nos spécimens (146-170 $\mu \mathrm{m}$ à frais), mais le corps interne est long (16-18 $\mu \mathrm{m}$, au lieu de 10-12 $\mu \mathrm{m})$, et la cellule R1 est petite ; en outre, la + a un ovéjecteur long $(2000 \mu \mathrm{m}$ chez le spécimen mesuré, au lieu de 350 à $950 \mu \mathrm{m}$ chez nos spécimens) (in Bain, 1980).

Notre matériel constitue donc une espèce nouvelle, Eufilaria kalifai n. sp., dédiée à M. Kalifa en remerciement de l'aide apportée pour la capture des pies.

\section{II - Développement d'Eufilaria kalifai n. sp. chez Culicoides nubeculosus}

La microfilarémie ( $f g .5$ ) dans le sang périphérique (veine brachiale) est presque nulle à midi et atteint un maximum de 50 à 100 microfilaires pour $10 \mu \mathrm{l}$, selon les pies observées, à 3 heures du matin (heure solaire).

Les Culicoïdes sont gorgés sur la pie pendant la phase montante de la microfilarémie, entre $21 \mathrm{~h}$ et $0 \mathrm{~h}$; ils sont conservés à $25^{\circ}$. Les premiers stades infestants apparaissent huit jours plus tard ; la plupart des larves ont terminé leur développement le $10^{\mathrm{e}}$ jour. Sur un total de 73 Culicoïdes gorgés, $36 \mathrm{~L}_{3}$ sont récoltées.

\section{Morphologie de la larve infestante (fig. 3 et 4)}

Larve d'environ un demi-millimètre de long, amincie dans la région antérieure. Tête arrondie ; papilles labiales externes petites; papilles céphaliques bien visibles ; amphides à la hauteur de ces papilles; cavité buccale à peine plus longue que large et à base à peine cuticularisée; œesophage à portion musculaire à peu près aussi longue que la glandulaire; celle-ci ne se distingue de l'intestin que par la présence de l'axe cuticulaire longitudinal. Jonction œsophage-intestin en général à mi-corps, parfois un peu en arrière ; déchets métaboliques accumulés dans la région postérieure de l'intestin ; cellule excrétrice grande, à pore souvent réfringent; ébauche génitale

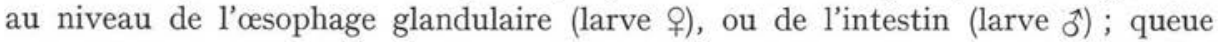
cylindrique, à extrémité arrondie ornée par 4 courtes languettes subterminales; les latérales sont arrondies et portent les phasmides (visibles sur certains spécimens); les médianes sont arrondies ou coniques.

Dimensions :

Corps long de 440 à $580 \mu \mathrm{m}$ et large de 13 à $15 \mu \mathrm{m}$; œsophage long de 210 à $310 \mu \mathrm{m}$, avec portion musculaire de 80 à $205 \mu \mathrm{m}$; pore excréteur à $96-116 \mu \mathrm{m}$ de l'apex; queue longue de 17 à $22 \mu \mathrm{m}$. Pour une larve + , longue de $580 \mu \mathrm{m}$ et large de $13 \mu \mathrm{m}$, cavité buccale haute de $3,5 \mu \mathrm{m}$, anneau nerveux et pore excréteur à 80 et

I. Supperer cite en outre Garrulus glandarius parmi les hôtes de cette espèce. Il n'a pas fixé d'holotype. Nous désignons pour celui-ci un spécimen femelle parasite de Turdus viscivorus. En effet, les microfilaires ayant une importance diagnostique particulière, un spécimen $q$ nous parait plus caractéristique et $T$. viscivorus est le premier nom cité dans la liste d'hôtes. 


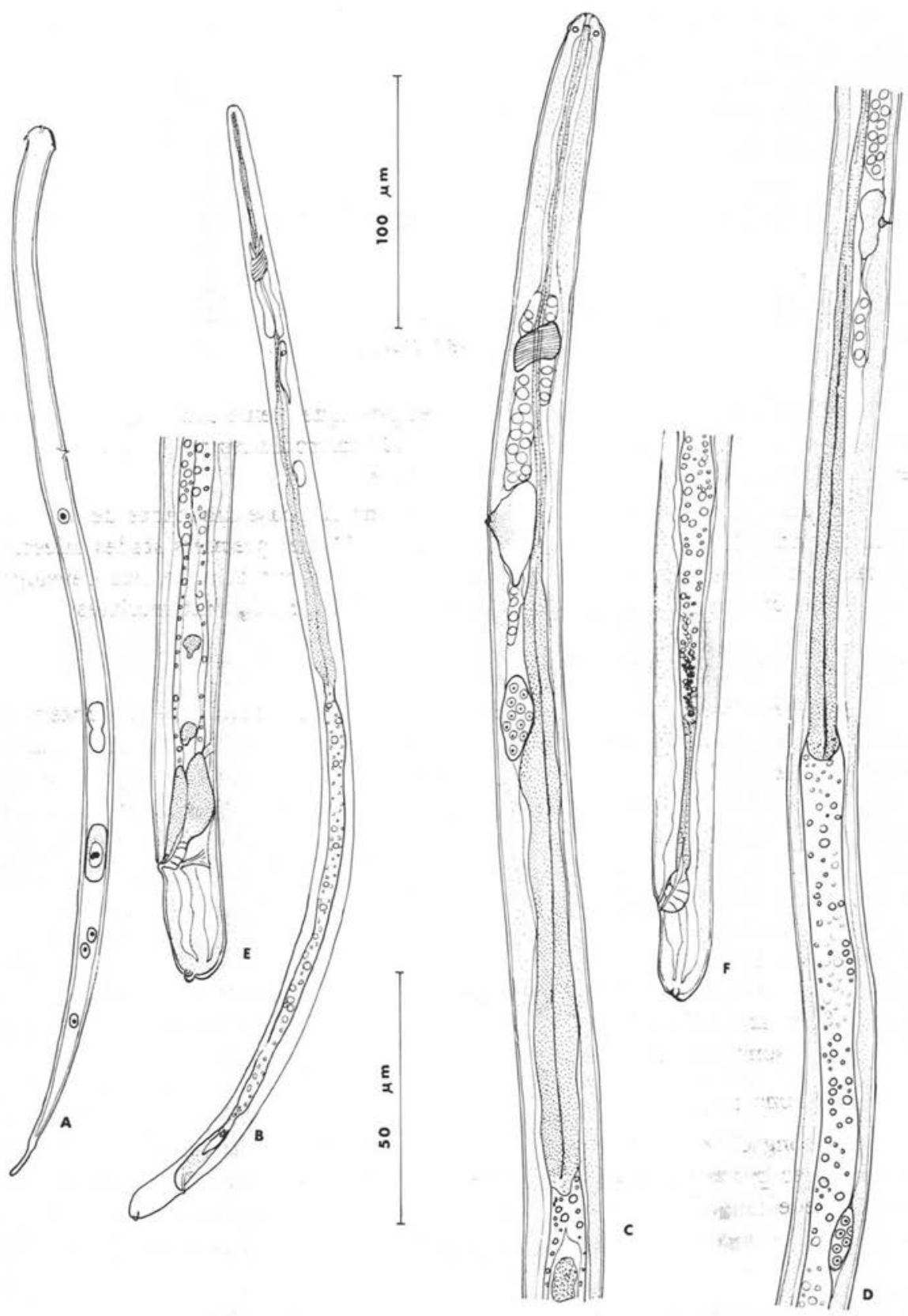

FIG. 3. - Eufilaria kalifai n. sp.; A : microfilaire sanguine en coloration vitale (pie 4I9 DS); B : stade infestant; $C$ : région antérieure d'une larve ; ; $D$ : position de l'ébauche génitale chez une larve ${ }^{*} ;$ E et $F: 2$ régions postérieures, vue latérale. (B, éch. Ioo $\mu \mathrm{m}$; le reste, éch. $50 \mu \mathrm{m}$.) 


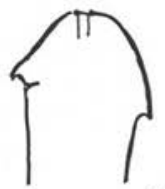

A<smiles>C1CCCCCC1</smiles>

B
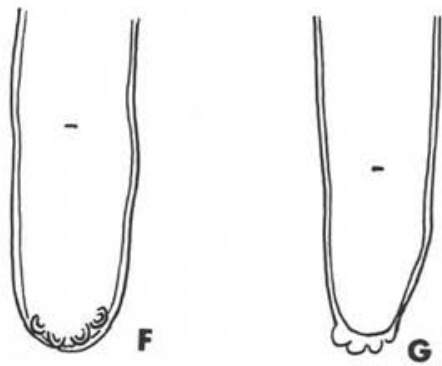

$\mathbf{F}$

G

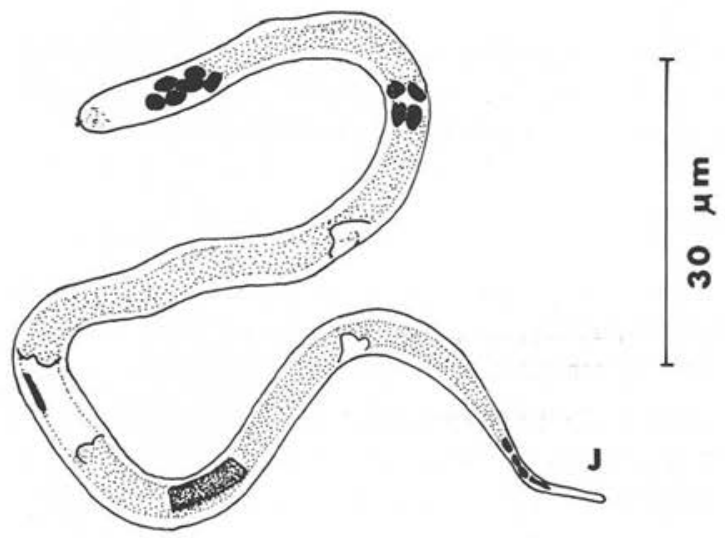

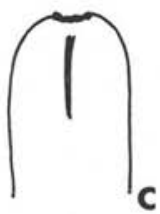<smiles>CCCCCCCCCCCCC</smiles>

D

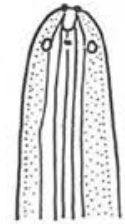

E
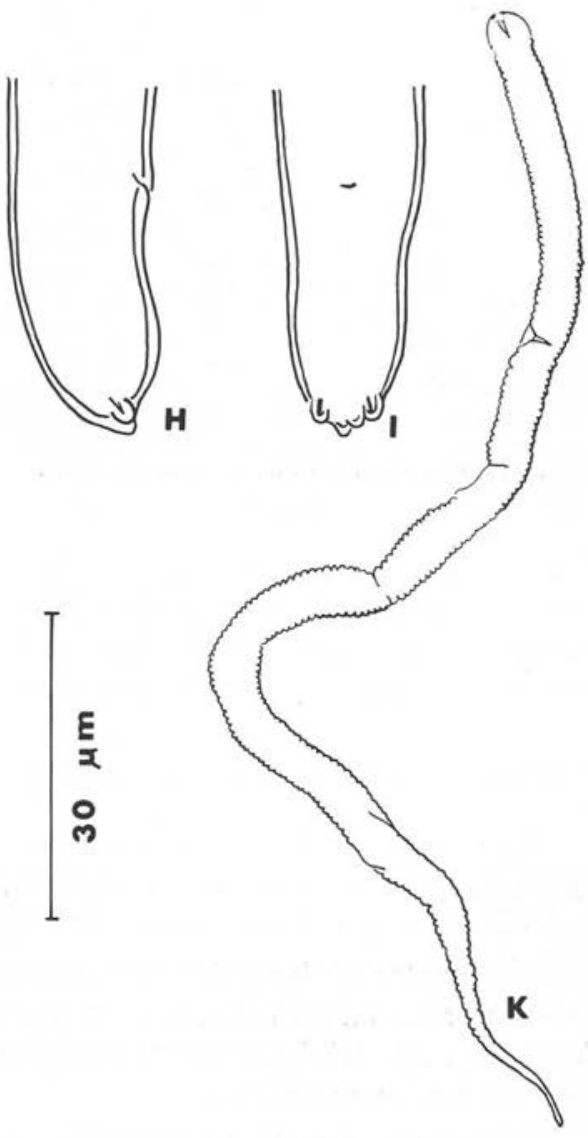

Fig. 4. - Eufilaria kalifai n. sp.; A à D, tête de la microfilaire ; A à C : à frais, en différentes positions : crochet vu de profil (A), de face (B), côté opposé au crochet (C); D : après fixation dans l'utérus, crochet vu de profil ; E : tête du stade infestant, vue latérale ; F et G : queue de 2 larves infestantes, vue ventrale; $\mathrm{H}$ et I : queue d'une $3^{\mathrm{e}}$ larve, vues latérale et ventrale ; $\mathrm{J}$ : microfilaire sanguine colorée au Giemsa ; $\mathrm{K}$ : microfilaire extraite de Yutérus de la $O$ holotype. (A à D, à main levée ; le reste, éch. $30 \mu \mathrm{m}$. 


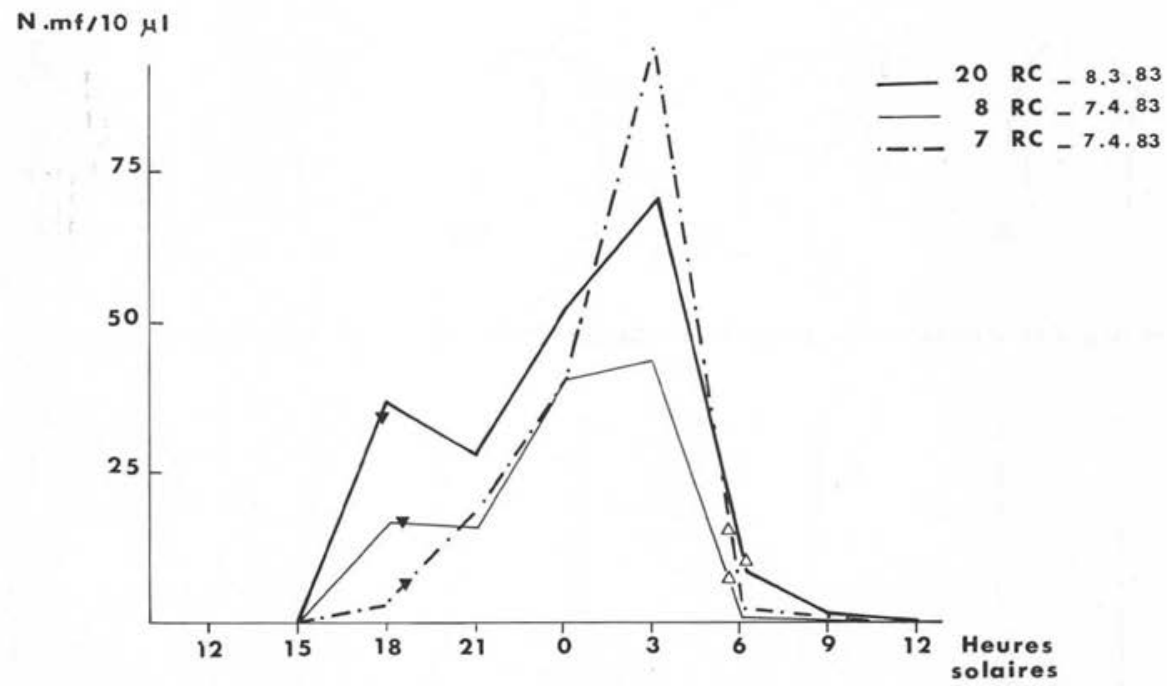

FIG. 5. - Variation de la microfilarémie au cours d'un nycthémère ( 8 au 9 mars 1983) chez les pies $7 \mathrm{RC}, 8 \mathrm{RC}$ et $20 \mathrm{RC}$. Chaque point représente le nombre de microfilaires observées dans $40 \mu$ l de sang prélevé à la veine brachiale. (n. mf. = nombre de microfilaires.) Les flèches indiquent le lever et le coucher du soleil.

$110 \mu \mathrm{m}$ de l'apex, œsophage long de $310 \mu \mathrm{m}$ (portion musculaire longue de $205 \mu \mathrm{m}$ ), rectum long de $27 \mu \mathrm{m}$; queue longue de $22 \mu \mathrm{m}$; ébauche génitale à $160 \mu \mathrm{m}$ de l'apex. Chez une larve $\widehat{\sigma}$ de $550 \mu \mathrm{m}$ de long, ébauche génitale à $310 \mu \mathrm{m}$ de l'apex.

Comparaison avec les autres cycles d'Eufilaria

On connaît les développements larvaires de 3 espèces : E. longicaudata (cf. Hibler, 1963), E. delicata et E. bartlettae (cf. Bain, 1980), qui, du point de vue de la morphologie des adultes, sont les espèces les plus proches d'E. kalifai (cf. discussion).

Les quatre cycles présentent une forte homogénéité : les vecteurs sont des Culicoides; les larves infestantes sont courtes $(<600 \mu \mathrm{m})$ et minces $(13-15 \mu \mathrm{m})$; l'œsophage est divisé et se termine à mi-corps ${ }^{2}$; la queue est une fois et demi à deux fois plus longue que large.

L'extrémité caudale présente deux types : elle est simplement arrondie et lisse chez les 2 espèces parasites de Turdidae, E. delicata et $E$. bartlettae, et porte 4 petites languettes chez les parasites de Corvidae, E. longicaudata et E. kalifai.

2. Selon Hibler ( 1963$)$, l'œsophage d'E. longicaudata ne mesure que roo-140 $\mu \mathrm{m}$ et est uniquement musculaire; la portion glandulaire a vraisemblablement, dans ce cas, été confondue avec l'intestin. 


\section{Conclusion}

Pendant longtemps en région holarctique, les seules Eufilaria connues étaient en Europe E. delicata Supperer, 1958, chez les Turdidés (et peut-être chez les Corvidés) et en Amérique du Nord E. longicaudata Hibler, 1963 chez les Corvidés.

Cette note et la récente publication de l'un d'entre nous (Bain, 1980) montrent que le genre Eufilaria est probablement très diversifié. Les microfilaires fournissent parfois le seul caractère discriminatif.

Les formes infestantes présentent également des caractères distinctifs : en effet, les 2 espèces parasites de Corvidés s'opposent aux 2 espèces parasites de Turdidés par la morphologie de la queue : languettes présentes dans le premier cas, absentes dans le second.

\section{BIBLIOGRAPHIE}

Anderson R. C., Bain O. : Keys to genera of the order Spirurida. Part 3. Diplotriaenoidea, Aproctoidea and Filarioidea. In CIH Keys to the Nematode Parasites of Vertebrates, R. C. Anderson, A. G. Chabaud, S. Willmott (eds), Commonwealth Agricultural Bureaux, Farnham Royal, Bucks, England, r976.

Anderson R. C., Bennetr G. F. : Eufilaria mcintoshi, n. sp. from Padda oryzivora (L.). Proc. helminth. Soc. Wash., 1960, $27, \mathrm{I}_{3} \mathrm{I}^{-1} \mathrm{I}_{5}$.

Anderson R. C., Prestwood A. K. : Singhfilaria hayesi n. sp. from Meleagris gallopavo and a revision of Lemdaninae (= Eufilariinae). Can. J. Zool., 1969b, 47, 1015-1019.

Annetr H. E., Dutron J. E., Elliot J. H. : Report of the malaria expedition to Nigeria of the Liverpool School of Tropical Medicine and Medical Parasitology. Part II. Filariasis. Thompson Yates and Johnson Lab. Rep., I $90 \mathrm{I}, 4, \mathrm{I}-92$.

Barn O. : Deux Filaires du genre Eufilaria chez le Merle : développement chez Culicoides nubeculosus. Ann. Parasitol. Hum. Comp., 1980, s5, 583-59o.

Bartletr C. M. : Filarioid nematodes (Filarioidea: Onchocercidae) of the crow (Corvus brachyrhynchos brachyrhynchos Brehm) in Southern Ontario. These, Master of Science, Univ. Guelph, 1979, I55 p. polycopiées.

Deshmukn P. G. : Two new species of a new genera Neofilaria from Indian quails. Riv. Parassit., I $968,29,123-128$.

Hrbler C. P. : Onchocercidae (Nematoda : Filarioidea) of the American magpie, Pica pica hudsonia (Sabine), in northern Colorado. Ph. D. thesis, Colorado State University, Ft. Collins Colorado, I963, I89 p. polycopiées.

Seurat L. G. : La filaire du moineau. Bull. Soc. Hist. Nat. Afr. N., I921, I2, 28-3I.

SINGH B. S. N. : Studies on the helminth parasites of birds in Hyderabad State. Nematoda IV. J. Helminthol., 1949, 23, 39-56.

Sonin M. D. : [A new genus of Nematodes - Eufilariella Sonin (Filariata, Aproctoidea), Parasitizing Birds]. Materialy $k$ nauch. konf. Vses, obshchestva gel'mintol., $1965,1,202-203$.

Sultana A. : New species of Chandlerella Yorke and Maplestone, 1926 and Splendidofilaria Skrjabin, 1923 from Indian birds with a discussion of related genera. J. Parasitol., 1962, 48, 707-714.

SUPPERER R. : Zwei neue Filarien (s.1.) Eufilaria delicata spec. nov. und Ornithofilaria böhmi spec. nov. aus der Misteldrossel, Turdus viscivorus L. Z. Parasitkde, 1958, I 8, 312-319. 